\title{
THE SMOOTHING OF THE ORIENTATION DISTRIBUTION DENSITY INTRODUCED BY CALCULATION METHODS
}

\author{
JAN POSPIECH \\ Polish Academy of Sciences, Aleksander Krupkowski Institute of Metallurgy and \\ Materials Science, 30-059 Krakow, ul. Reymonta 25
}

(Received 10 December 1995)

The values of the orientation density distribution function (ODF) depend as a rule on the applied calculation method. In the series expansion method the calculation result is influenced by truncation of the series. With decreasing of the expansion order $l_{\max }$ the width of smoothing around the ODF maximum increases and its height decreases.

It is shown that the smoothing of the orientation density caused by series truncation can be described analytically by a Gaussian type function. Disturbing undulations of negative and positive values around ODF maxima characteristic for harmonic expansion can be suppressed by using additional smoothing functions.

KEY WORDS: Series truncation, ODF-smoothing, single orientation, Gaussian distribution.

\section{INTRODUCTION}

The basic question in texture analysis is the reliability of the ODF calculated from experimental data. The values of the ODF depend, in general, on the kind and quality of experimental data and are also influenced by the kind and particulars of the applied calculation method.

In the present paper the smoothing phenomenon in the ODF calculated from single orientations will be analysed.

If different methods are used (series expansion or direct) in the calculations of the ODF with the same input data (here a single orientation set), one can expect that the preferred orientations in the ODF (texture components) are located in the same positions, but the relative heights of the maxima in these positions may be different. The latter is caused by the data smoothing specific for each of the methods. The width of the peak of the orientation density attributed to an orientation will be assumed as the measure of smoothing. On account of normalization the height of the peak decreases with the increase of its width.

The series expansion method introduces smoothing (around each orientation) through the effect of truncation of the series at $l_{\max }$ and additionally also by discretization. The calculations of the ODF $f(g)$ are performed using the known expression of series expansion by Bunge (1982)

$$
\mathrm{f}(\mathrm{g})=\frac{1}{J} \sum_{l=0}^{l_{\max }}(2 l+1) \sum_{m n} \sum_{j=1}^{J} \mathrm{~T}_{l}^{{ }^{*} \mathrm{mn}}\left(\mathrm{g}_{j}\right) \mathrm{T}_{l}^{\mathrm{mm}}(\mathrm{g})
$$


or in the discrete ODF-space

$$
\mathrm{f}(\mathrm{g})=\sum_{l=0}^{l_{\max }}(2 l+1) \sum_{m n} \sum_{i=1}^{I}\left(\frac{k_{i}}{J}\right) \mathrm{T}_{l}{ }^{{ }^{\mathrm{mn}}}\left(\mathrm{g}_{i}\right) \mathrm{T}_{l}^{\mathrm{mm}}(\mathrm{g})
$$

where $J$ is the total number of measured orientation $g_{j} . I$ is the number of cells and $k_{i}$ is the number of orientations falling into the $i$-th cell $\Delta g_{i}$ with the center $g_{i}$ in the discretizised orientation space.

The greater $l_{\max }$, the smaller is the width $\psi$ (of smoothing) of the peak of the orientation density around an orientation. Simultaneously, with the increase of $l_{\max }$ the disturbing oscillations of negative and positive values around a density peak (known for harmonics) become (in their absolute scale) larger. For infinite $l_{\max }$ the density function assumes the form of a delta peak.

In the direct method the smoothing is introduced by discretization of the orientation space and the result (the ODF) obtained according to the formula

$$
\mathrm{f}\left(\mathrm{g}_{i}\right)_{d}=\frac{k_{i}}{J \Delta g_{i}}
$$

has the form of a histogram.

Another form of smoothing in both methods can be the additional assigning of a smoothing function, e.g. Gauss-type distribution

$$
\mathrm{S}(\phi, \omega)=\mathrm{S}_{0} \exp \left(-\phi^{2} / \omega^{2}\right)
$$

to each orientation or to each cell in the discrete orientation space.

If the ODF $\mathrm{f}(\mathrm{g})$ of a superposition of Gauss-type distributions (3) is represented by series expansion, then (Pospiech and Lücke 1975)

$$
\mathrm{f}(\mathrm{g})=\sum_{l=0}^{l_{\max }} \frac{\exp \left(-l^{2} \omega^{2} / 4\right)-\exp \left(-(l+1)^{2} \omega^{2} / 4\right)}{1-\exp \left(-\omega^{2} / 4\right)} \sum_{m n} \sum_{j=1}^{J} \mathrm{~T}_{l}{ }^{* \mathrm{mn}}\left(\mathrm{g}_{j}\right) \mathrm{T}_{l}^{\mathrm{mm}}(\mathrm{g}) .
$$

Through an appropriate choice of the width $\omega$ of the additional Gauss-type distribution in the series expansion (4) (see e.g. Wagner 1986) the known disturbing undulations around an ODF maximum can be suppressed. An example will be shown in this paper.

The size of the cell $d \approx(\Delta \mathrm{g}) \frac{1}{3}$ in the discrete ODF-space should correspond to the precision of the measurement and be a measure of the resolution of the ODF. The size $d$ from the point of view of series expansion should be then the lower limit for the width of smoothing $2 \psi$ caused by truncation at $\mathrm{L}=l_{\max }$ (when $\left.\omega=0\right)$. In an earlier paper by Pospiech (1987) it has been found that the expansion order $\mathrm{L}$ is connected with the size $d$ by the simple formula

$$
\mathrm{L}=\frac{360^{\circ}}{d \sqrt{2}} .
$$

In the present paper the effect of series truncation of the calculated orientation density around a single orientation is analysed. An analytical form for the description of the smoothing of orientation density caused by truncation is presented and also the influence of smoothing functions additionally introduced into the series is discussed. In the analytical derivation of the smoothing of orientation density caused by truncation the relation (5) is proved. 
THE EFFECT OF TRUNCATION OF SERIES EXPANSION ON THE FORM OF THE ORIENTATION DENSITY (ODF) AROUND A SINGLE ORIENTATION

The orientation density around one single orientation expressed by series expansion has according to the expansion (1a) the form

$$
\mathrm{f}(\mathrm{g})=\sum_{l=0}^{l_{\max }}(2 l+1) \sum_{m n} \mathrm{~T}_{l}^{{ }^{*} \mathrm{mn}}\left(\mathrm{g}_{j}\right) \mathrm{T}_{l}^{\mathrm{mm}}(\mathrm{g})
$$

For simplification the orientation $g_{j}$ will be located in the origin of the coordinate system $g_{j}=(0,0,0)$. Then there is $\mathrm{T}_{l}{ }^{{ }_{\mathrm{mnn}}}(\mathrm{e})=\delta_{\mathrm{mn}}$ and Exp. (6) transforms into $\mathrm{f}(\mathrm{g})=$ $\sum_{l=0}^{\llcorner}(2 l+1) \sum_{m} \mathrm{~T}_{l}{ }^{*} \mathrm{~mm}(\mathrm{~g})$. The form of the density scattering around the orientation depends only on the orientation distance $\phi$ defined by the trace of the orientation matrix. According to Bunge (1982) this lead to the expression

$\mathrm{f}(\phi)=\sum_{l 0}^{L}(2 l+1) \sum_{m=-1}^{+1} \mathrm{e}^{\mathrm{i} m \phi}=2 \sum_{l=0}^{L}(2 l+1)\left[\sum_{m=0}^{l} \cos m \phi-\frac{1}{2}\right]$.

After summing over $s$ one obtains the form

$$
f(\phi)=\sum_{l=0}^{L}(2 l+1)\left[\frac{2 \cos l \frac{\phi}{2} \sin (l+1) \frac{\phi}{2}}{\sin \frac{\phi}{2}}-1\right]
$$

describing the smoothing of the orientation density around an orientation which is introduced by truncation of the series at the order $l_{\max }=\mathrm{L}$. For $\phi=0$ one gets the maximum value $H_{0}(L)=f(\phi)_{\max }$ (i.e. the height of the peak)

$$
\mathrm{H}_{0}(\mathrm{~L})=\mathrm{f}(\phi \rightarrow 0)=\sum_{l=0}^{L}(2 l+1)^{2} \cong \mathrm{L}\left[\frac{3}{4}(\mathrm{~L}+1)(\mathrm{L}+2)+1\right]+1
$$

depending on the expansion order $\mathrm{L}$. For $\mathrm{L} \rightarrow \infty$ there is $\mathrm{H}_{0}(\mathrm{~L}) \rightarrow \infty$ and $\mathrm{f}(\phi)$ takes the form of a delta peak.

\section{THE APPROACH TO A GAUSSIAN TYPE DISTRIBUTION}

It has been found that the form of the orientation density (the smoothing) around an orientation caused by truncation of the series expansion can be approximated analytically by the following form of a Gaussian-type function.

$$
S(\phi, \psi)=S_{0}(\psi) \exp \left(-\frac{\phi^{2}}{2 \psi^{2}}\right)
$$

with such normalization that the height is expressed by

$$
S_{0}(\psi)=\frac{\sqrt{4 \pi}}{\psi\left(1-\exp \left(-\psi^{2 / 4}\right)\right)} \approx \frac{8 \sqrt{\pi}}{\psi^{3}}
$$

where $\alpha=\psi \sqrt{2}$ is the width at the 1/e height of the Gauss-type peak. Expression (9) differs from the known form of the Gaussian type distribution normalized to one in 
the ODF-space (Pospiech, Lücke (1975)) only by the value 2 in the denominator of the exponent of the distribution, the expression (10) for the height of the peak remaining unchanged.

\section{RELATION BETWEEN THE EXPANDING ORDER L AND THE WIDTH $\Psi$ OF SMOOTHING.}

The analytical relation between $\mathrm{L}$ and $\psi$ can be found by comparing the expression (8) and (10)

$$
\mathrm{H}_{0}(\mathrm{~L})=\mathrm{S}_{0}(\psi) \text {. }
$$

This comparison leads to the following equation of the 3rd degree

$$
\frac{4}{3} \mathrm{~L}^{3}+4 \mathrm{~L}^{2}+\frac{11}{3} \mathrm{~L}+\left(1-\mathrm{S}_{0}(\psi)\right)=0 \text {. }
$$

Assuming according to (10) the simplified form $S_{0}(\psi)=8 \sqrt{ } / \psi^{3}$ one gets the following well approximated solution

$$
\mathrm{L}=\frac{\pi}{\psi \sqrt{2}}-1
$$

which represents the relation between the expanding order $\mathrm{L}$ and the width of smoothing $\alpha=\sqrt{2} \psi$. Eq (13) has the form of the formula (5) $L=2 \pi /(d \sqrt{2})$ (earlier derived by Pospiech (1987)) where $d$ was assumed as the measure of the resolution power and is here expressed in arc measure. The width $\alpha$ of smoothing is the greater, the smaller the expansion order $\mathrm{L}$.

Introducing the expression $\psi=\pi /((L+1) \sqrt{2})$ obtained from (13) into the modified Gauss-type function (9) one gets the analytical form of the smoothing $S(\phi, L)$ in the near surroundings of each orientation describing the effect of truncation in dependence of the expansion order L. After taking into account the simplification (10) this expression for smoothing $S(\phi, L)$ has the following form

$$
\mathrm{S}_{0}(\phi, \mathrm{L})=\frac{2\left(2 \pi^{7}\right)^{1 / 2}}{(L+1)^{3}} \exp \left(-\frac{\phi^{2}(L+1)^{2}}{\pi^{2}}\right) .
$$

The result of the comparison of the orientation densities around a single orientation calculated by series expansion (6) and by the formula (14) in dependence of the expansion order $L$ is shown in figure 1 .

The plot on figure 1 demonstrates that the derived formula (14) approximates very well the form of smoothing caused by series truncation. The characteristic undulations of negative and positive values occurring for the harmonic expansion are visible in the figure. Through these undulations in the surroundings of each single orientation the ODF calculated by using series is distorted and will show some differences in comparison with the ODF created by superimposing Gaussian type functions (14). This is demonstrated, by an example (in Figure 2) in which ODF profiles from series expansion (up to $\mathrm{L}=34$ ) and from the corresponding superimposed Gaussian functions (14) are plotted for a set of four orientations. The orientations are situated at points distanced at $\Delta \mathrm{x}=8^{\circ}$ and $12^{\circ}$ from each other (from left to right). To the expansion order $\mathrm{L}=34$ there corresponds according to (13), the width $\alpha=\sqrt{2} \psi=5.15$. Two 


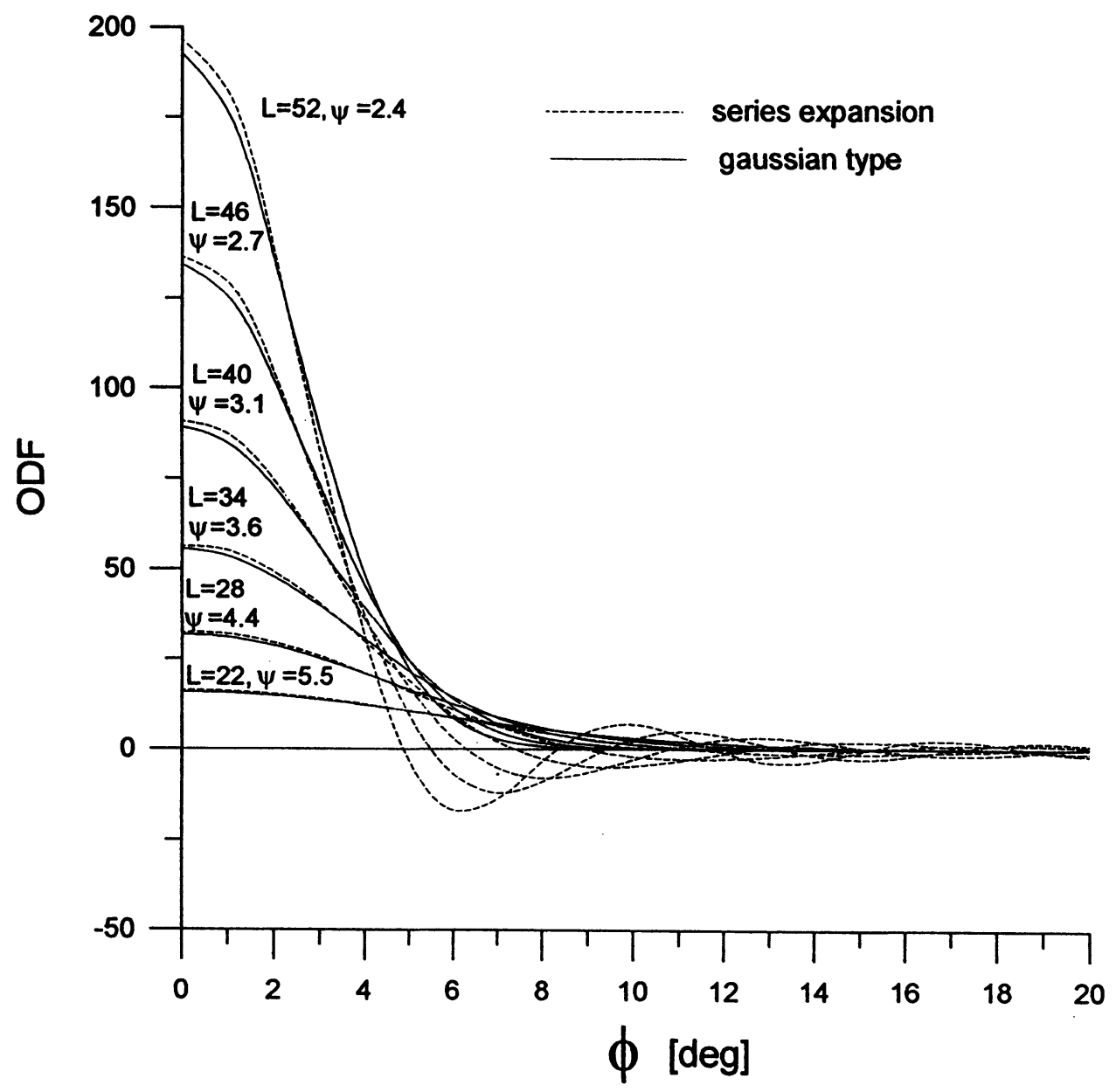

Figure 1 The smoothing of the orientation density around one orientation caused by truncation of the series at $l_{\max }=\mathrm{L}$ compared with a Gaussian type function.

peaks are separated if the distance between them is $\Delta \mathrm{x}_{0}>\sqrt{2} \alpha=7.28$. Each distance $\Delta \mathrm{x}$ is greater than $\Delta \mathrm{x}_{0}$, thus all peaks should be separated from each other. It is easily seen in figure 2 that this is fulfilled for the case of superposition of Gaussian functions (Eq. (9)), whereas in the case of series expansion the two first peaks are not clearly separated. On figure 2 other deviations can be also observed.

THE INFLUENCE OF A GAUSSIAN FUNCTION INTRODUCED INTO THE SERIES ON THE FORM OF SMOOTHING

Let assume that each orientation of the set is represented in a smoothed form described by the Gaussian distribution (Eq. (3)) with the width $\omega$. If the ODF is determined from 


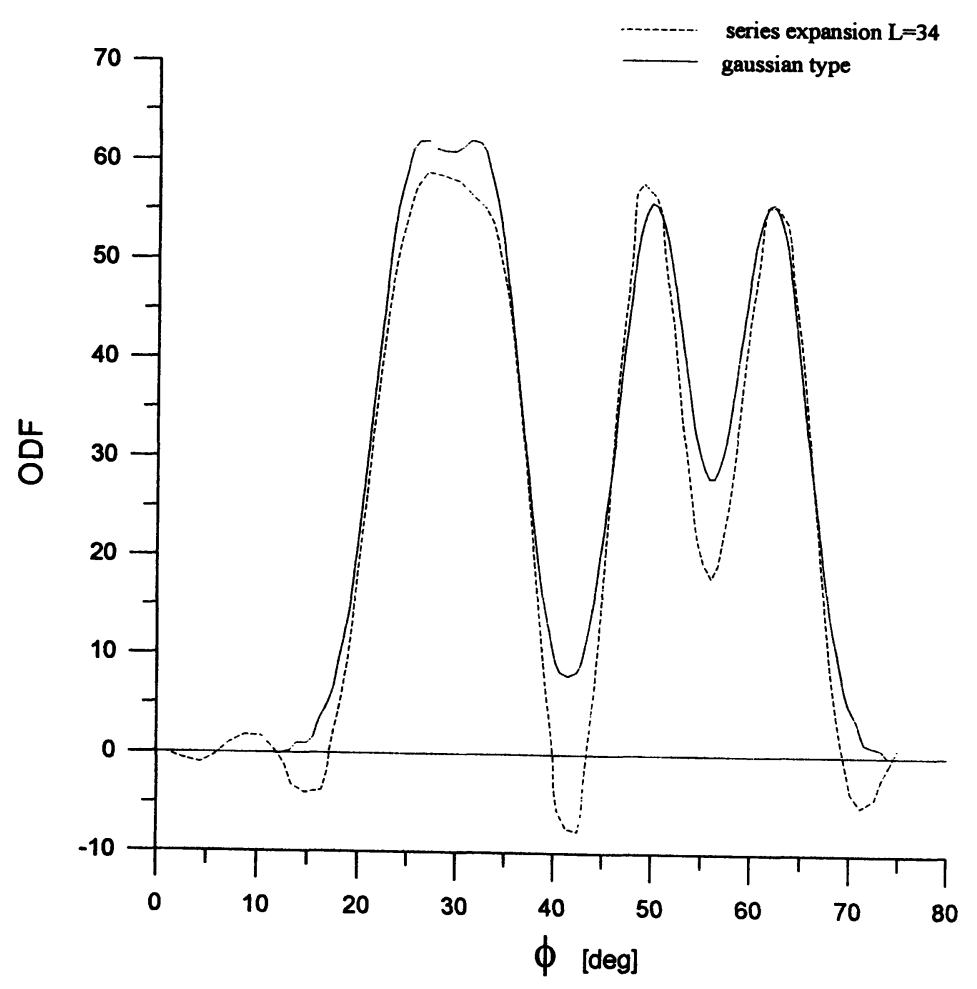

Figure 2 Profiles of ODF values calculated for a set of four orientations by series expansion up to 34 (dotted lines) and by the direct method (continuous lines) with appropriate Gaussian type function.

such a set by using series expansion, the known expression (4) is applied. The consideration can be limited to one dimension and to one orientation (i.e. one Gaussian distribution), and then proceeding similarly as in the case of deriving function (7) one gets for ODF:

$$
f(\phi, L, \omega)=\sum_{l=0}^{L} S(\omega)\left[\frac{2 \cos l \frac{\phi}{2} \sin (l+1) \frac{\phi}{2}}{\sin \frac{\phi}{2}}-1\right]
$$

and the height of the maximum is equal to

where

$$
f(\phi=0, L, \omega)=\sum_{l=0}^{L}(2 l+1) \cdot S(\omega)
$$

$$
S(\omega)=\frac{\exp \left(-l^{2} \omega^{2} / 4\right)-\exp \left(-(l+1)^{2} \omega^{2} / 4\right)}{1-\exp \left(-\omega^{2} / 4\right)}
$$

The smoothing function $S(\omega)$ (with the width $\omega$ ) introduced into the series determines (with the existing influence of the series truncation) the form of smoothing around the orientation. With increasing $\mathrm{L}$ it displaces out, to a greater and greater extent, the smoothing resulting from the series truncation, simultaneously eliminating the characteristic undulations of negative and positive values around the maximum. 
For a sufficiently great truncation order $\mathrm{L}$, depending on the adapted smoothing width $\omega$, the parameters defining the form of smoothing around the orientation become stabilized and take constant values with arbitrarily small accuracy depending on L.

The course of the stabilization of the smoothing around the orientation is illustrated by the curve in figure 3 (for the width $\omega=3,4,5,6,7$ ). They describe the increments of the height of maxima in dependence on the expansion order $L$. They have characteristic maxima and ranges of convergence to zero, which are shifted toward smaller values of $L$ with increasing width $\omega$ of the Gaussian distribution. The attaining of the convergence range of the curve is an indication that the shape of smoothing around the orientation is determined by the function $S(\omega)$ and also that the undulations of negative and positive values around the orientation will disappear. The position of the maximum indicates the beginning of the growing domination of the smoothing $S(\omega)$ around the orientation over the form of the smoothing caused by truncation. Figure 4 shows additionally how the absolute value of the height of the peak changes for $\omega=5$. For $L=60$ it attains practically the range of stabilization. The width of the smoothing not shown here changes in a similar way.

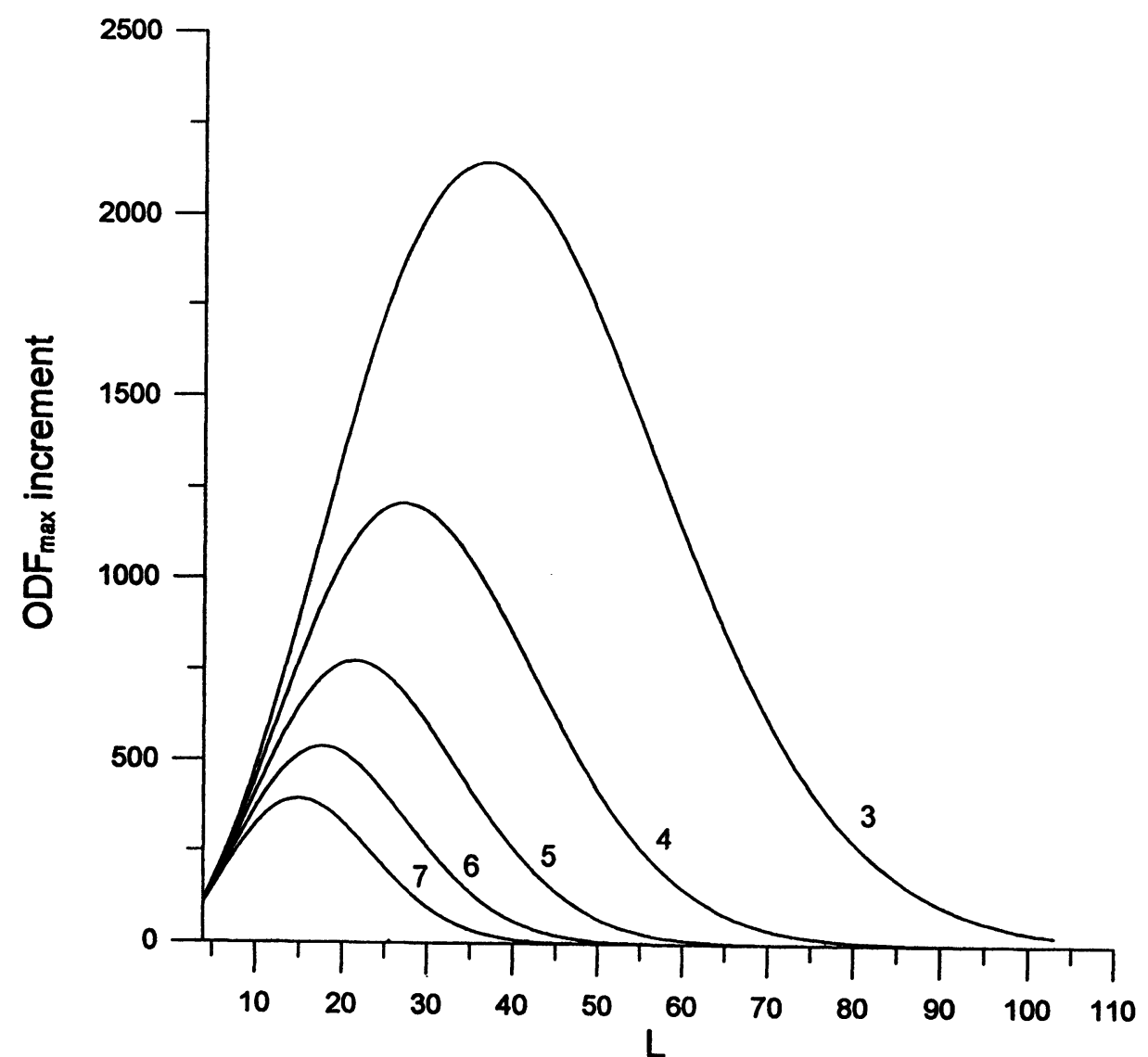

Figure 3 Increments of maxima values of OD densities around one orientation introduced into the series expansion in the form of a Gaussian type function for various width $\omega=3,4,5,6,7$ degrees. 




Figure $4 \mathrm{ODF}$ maximum of a Gaussian type function with the width $5 \mathrm{deg}$. introduced into the series expansion in dependence of the truncation range $L$.

\section{CONCLUDING REMARKS}

When in the calculations of the orientation density (ODF) from a set of single orientations the direct method is used, then there are some possibilities to control the systematic errors induced by the method. The measured single orientations are then introduced directly into the orientation space. The accuracy of the measurement is then taken into account through proper selection of the size of the cell in the discrete orientation space. For smoothing the calculation results (having the form of a histogram) scattering functions (e.g. in the form of a Gaussian distribution) are additionally used.

In the calculation results of orientation distribution densities based on series expansion there appear the effect of series truncation, as well as disturbing oscillations of the harmonics. They bring about definite smoothing around preferred orientations with areas of negative and positive pulsations. These make difficult the controlling of the calculation results and make their interpretation uncertain. This refers in particular to the results at low value levels (below $10 \%$ of maximum) which often cannot be accepted as reliable. As it is shown, the smoothing introduced through the series expansion method can be described analytically as a function dependent on the expansion order $L$ in the form of a Gaussian distribution. The undulations of harmonics occurring around the preferred maxima can be suppressed by introducing an additional smoothing into the series. 
The quantitative description presented in this paper, enables a direct comparison of the calculation results obtained using the series expansion method with the superposition of the Gaussian distribution of the direct method. It permits also to link the series expansion order $\mathrm{L}$ with the measure of the resolution power associated with the measurement accuracy.

\section{Acknowledgements}

The author would like to thank J. Jura for valuable comments on the manuscript.

\section{References}

Bunge, H. J. (1982). Texture Analysis in Materials Science, Butterworths, London.

Pospiech, J. and Lücke K. (1975). The Rolling Textures of Copper and $\alpha$-Brasses Discussed in Terms of the Orientation Distribution Function. Acta Metallurgica, 997

Pospiech, J. (1987) Basic Relation for Comparison of Different ODF calculation Methods. In: Theoretical Methods of Texture Analysis. Ed. H. J. Bunge DGM Informationsgesellschaft, Oberursel, p. 95.

Wagner, F. (1986) Texture Determination by Individual Orientation Measurements. In: Experimental Techniques of Texture Analysis. Ed. H. J. Bunge. DGM Informationsgesellschaft. Oberursel, p. 115. 